\title{
A multicomponent exercise program's effects on health-related quality of life of institutionalized elderly
}

\begin{abstract}
Objective: To determine the effects of a multicomponent exercise program on health-related quality of life (HRQOL). Design: A quasi-experimental pretest-posttest design was used in the study. Setting: Participants were recruited from the public-funded shelter home located in Seremban, Negeri Sembilan, Malaysia. Participants: The experimental group consisted of 27 volunteers older than 60 years who performed 60 minutes of aerobic, strength, flexibility, and balance training 3 times a week for 12 weeks, supervised by a physiotherapist. The control group consisted of 25 volunteers who continued with their current level of activity. Main outcome measures: Physical health component summary (PCS) and mental health component summary (MCS) of the 12-Item Short Form Health Survey HRQOL. Results: The exercise group increased in the level of PCS and MCS by $8.4 \%(\mathrm{P}<.05)$ and $2.9 \%(\mathrm{P}>.05)$, respectively. The control group shows decline in PCS and MCS by $9.3 \%(\mathrm{P}<.05)$ and $6.8 \%$ $(\mathrm{P}>.05)$, respectively. Conclusions: This study suggests that a multicomponent exercise program may improve HRQOL among institutionalized elderly.
\end{abstract}

Keyword: Institutionalized older persons; Malaysia; Mental health; Multicomponent exercise; Physical health; Quality of life 